\title{
A PROOF OF PARISI'S CONJECTURE ON THE RANDOM ASSIGNMENT PROBLEM
}

\author{
SVANTE LINUSSON AND JOHAN WÄSTLUND
}

\begin{abstract}
An assignment problem is the optimization problem of finding, in an $m$ by $n$ matrix of nonnegative real numbers, $k$ entries, no two in the same row or column, such that their sum is minimal. Such an optimization problem is called a random assignment problem if the matrix entries are random variables. We give a formula for the expected value of the optimal $k$-assignment in a matrix where some of the entries are zero, and all other entries are independent exponentially distributed random variables with mean 1 . Thereby we prove the formula $1+1 / 4+1 / 9+\cdots+1 / k^{2}$ conjectured by G. Parisi for the case $k=m=n$, and the generalized conjecture of D. Coppersmith and G. B. Sorkin for arbitrary $k, m$ and $n$.
\end{abstract}

\section{INTRODUCTION}

The problem of minimizing the sum of $k$ elements in a matrix of nonnegative real numbers under the condition that no two of them may be in the same row or column is called an assignment problem. A set of matrix positions no two in the same row or column is called an independent set. An independent set of $k$ matrix positions will also be called a $k$-assignment.

A random assignment problem, or RAP for short, is given by a number $k$, and an $m$ by $n$ matrix $(\min (m, n) \geq k)$ of random variables. If $P$ is a random assignment problem, we denote by $E(P)$ the expected value of the minimal sum of an independent set of $k$ matrix elements.

In this article we prove the following.

Theorem 1.1 (Parisi's Conjecture P98). Let $P$ be the RAP where $k=$ $m=n$ and the matrix entries are independent exponential random variables with intensity 1. Then

$$
E(P)=1+\frac{1}{4}+\frac{1}{9}+\cdots+\frac{1}{k^{2}} .
$$

We also prove the following two generalizations.

Theorem 1.2 (Conjectured by D. Coppersmith and G. B. Sorkin CS98). Let $P$ be an RAP where the matrix entries are independent exponential random variables with intensity 1 . Then

$$
E(P)=\sum_{i+j<k} \frac{1}{(m-i)(n-j)} .
$$

Date: 25th August 2018. 
Theorem 1.3 (Conjectured in [LW00]). Let $P$ be an RAP where some matrix entries are zero and all the other entries are independent exponential random variables with intensity 1 . Then

$$
E(P)=\frac{1}{m n} \sum_{i, j} \frac{d_{i, j}(P)}{\left(\begin{array}{c}
m-1 \\
i
\end{array}\right)\left(\begin{array}{c}
n-1 \\
j
\end{array}\right)},
$$

where $d_{i, j}(P)$ is an integer coefficient defined in terms of the combinatorics of the set of zeros, see Section 2.2.

The identity (2) will be referred to as the cover formula. When $P$ has no zeros,

$$
d_{i, j}(P)=\left(\begin{array}{c}
m \\
i
\end{array}\right)\left(\begin{array}{c}
n \\
j
\end{array}\right) .
$$

Hence Theorem $1.3 \Rightarrow$ Theorem 1.2. To see the implication Theorem 1.2 $\Rightarrow$ Theorem 1.1 (proved in CS98), note that if we put $m=n=k$ in (11), then the terms for which $\operatorname{gcd}(k-i, k-j)=d$ sum to $1 / d^{2}$ for $d=1, \ldots, k$.

As a consequence of Theorem 1.1 we also obtain a new and completely different proof of the following theorem, conjectured by M. Mézard and G. Parisi MP85.

Theorem 1.4 (D. Aldous A92, A01). Let $P$ be the RAP where $k=m=n$ and the matrix entries are independent exponential random variables with intensity 1. Then

$$
\lim _{k \rightarrow \infty} E(P)=\frac{\pi^{2}}{6}
$$

In Section 7 we mention some other corollaries of Theorem 1.3 .

1.1. Outline of the proof. A key result, proved in Section 4, is a formula for the probability that a nonzero row in an RAP is used in the optimal $k$-assignment, see Theorem 4.1] From [LW00] we know that the probability that a nonzero element in an RAP $P$ is used in the optimal $k$-assignment can be written $E(P)-E\left(P^{\prime}\right)$, where $P^{\prime}$ is obtained from $P$ by setting the matrix element in question to zero, see Theorem 2.2. Therefore, the formula for the probability that a row (or column) is used gives linear recursions for the values of the RAP's. Provided $m$ or $n$ is sufficiently large compared to $k$, this system of linear recursions has a unique solution given by the cover formula in Theorem [1.3, see Section [5. Finally in Section [6 we prove that for fixed $k$, fixed $m$, and a fixed set of zeros, $E(P)$ is given by a rational function in $n$, which must then agree with the cover formula.

1.2. Background. Random assignment problems have attracted the attention of researchers from physics, optimization, and probability. There are experimental results in O92, PR93. Constructive upper and lower bounds on $E(P)$ have been given in W79, O92, CS98, L93, K87, GK93. Mézard and Parisi MP85] used the non-rigorous replica method and arrived at the conjectured limit $\pi^{2} / 6$. This limit was subsequently established rigorously by Aldous A92, A01 using the weak convergence method on a weighted infinite tree model. In this paper we continue the exact formulas-approach inspired by [P98, and developed further in AS02, BCR02, CS98, CS02, LW00, EES01. 
There are also interesting results on similar problems such as finding a minimal spanning tree in a graph with random edge weights [BFM98, [FM89, F85, EES01. An intriguing question is why the Riemann $\zeta$-function appears in the limit of both the spanning tree and bipartite matching problems.

One tool that we believe can be useful to a wider range of problems is Theorem 2.2 below and its generalization Theorem 7.3 of [LW00].

\section{Preliminaries}

2.1. Probabilistic preliminaries. We say that a random variable $X$ is exponentially distributed with intensity $a$ if $\operatorname{Pr}(X>t)=e^{-a t}$ for $t \geq 0$. The intensity of $X$ is denoted $I(X)$. We have $E(X)=1 / I(X)$.

A random assignment problem $P$ is called standard if the matrix entries are either zero or independent exponentially distributed with intensity 1 . A standard RAP is determined by the numbers $k, m$ and $n$, and the set $Z$ of zero elements.

The following is a well-known lemma.

Lemma 2.1. Let $X_{1}, \ldots X_{n}$ be independent exponential variables with intensities $a_{1}, \ldots, a_{n}$ respectively. Then the probability that $X_{i}$ is minimal among $X_{1}, \ldots X_{n}$ is

$$
\frac{a_{i}}{a_{1}+\cdots+a_{n}} .
$$

The minimum is an exponential variable $Y$ of intensity $a_{1}+\cdots+a_{n}$ which is independent of which variable is minimal. Under the condition that $X_{i}$ is minimal, $X_{i}=Y$, and for $j \neq i$, we can write $X_{j}=Y+X_{j}^{\prime}$, where $X_{j}^{\prime}$ is exponential of intensity $a_{i}$, and the variables $Y$ and $X_{j}$ for $j \neq i$ are all independent.

We say that a deterministic assignment problem is generic if no sum of a set of nonzero matrix elements is equal to the sum of a different set of nonzero elements. In the RAP's that we consider, the distributions of the nonzero matrix elements are continuous. Hence all RAP's considered here will be generic with probability 1 . In the generic case, a nonzero element is used either in every optimal $k$-assignment, or in none. Hence we can without ambiguity speak of the probability that a certain nonzero element is used in the optimal $k$-assignment, without specifying whether we take this to mean some optimal $k$-assignment, or every optimal $k$-assignment.

Notice that this is not the case for zero elements. Even in a generic case, there may be several different optimal $k$-assignments, that differ in the choice of zero elements.

The following theorem, Theorem 2.10 of [LW00, is essential for the recursion equations in Section 5

Theorem 2.2. Suppose $P$ is a standard RAP. Suppose that the entry in position $(i, j)$ is not zero. Let $P^{\prime}$ be the standard $R A P$ where we have replaced the entry in position $(i, j)$ in $P$ with a zero. Then the probability that $(i, j)$ belongs to an optimal $k$-assignment in $P$ is $E(P)-E\left(P^{\prime}\right)$. 
2.2. Covers. We will consider sets of rows and columns in the $m \times n$-matrix. A set $\lambda$ of rows and columns is said to cover a set of zeros $Z$ if every matrix position in $Z$ is either in a row or in a column that belongs to $\lambda$. A cover with $s$ rows and columns will be called an $s$-cover. $k-1$-covers will be of particular importance. By a partial $k-1$-cover of $Z$, we mean a set of rows and columns which is a subset of a $k-1$-cover of $Z$.

By a cover of an RAP we mean a cover of its set of zeros. The cover coefficient $d_{i, j}(P)$ is the number of partial $k-1$-covers of $P$ with $i$ rows and $j$ columns. For this to be nonzero, $i$ and $j$ have to be nonnegative integers with $i+j<k$. It is convenient to regard the cover coefficient as well-defined, but zero, for integers $i, j$ outside this range.

We say that a set $\lambda$ of rows and columns is an optimal cover of $P$, if $\lambda$ covers $P$, and $\lambda$ has minimal cardinality among all covers of $P$. The following lemma is well-known. For a general introduction to matching theory we refer to LP86].

Lemma 2.3 (Lattice property of optimal covers). The set of optimal covers of a set of matrix positions forms a lattice, where one of the lattice operations is taking union of row sets and intersection of column sets, and the opposite lattice operation is taking intersection of row sets and union of column sets.

In particular, there is a row-maximal optimal cover containing every row that belongs to some optimal cover, and similarly a column-maximal optimal cover containing every column that belongs to some optimal cover.

An RAP can be reformulated in a setting of bipartite graphs with random weights on the edges. An assignment is then a matching. In this setting a cover of rows and columns is a vertex cover of the subgraph of edges with weight zero. We will borrow terminology from matching theory and speak of paths, etc. referring to the corresponding graph concepts.

The following two theorems were together with Theorem 2.2 our main tools for computing $E(P)$ recursively in [LW00].

Theorem 2.4. Let $A$ be a deterministic assignment problem. If there is no set of $k$ independent zeros, then each row and column which belongs to an optimal cover of the zeros must be used in every optimal k-assignment.

Let $c$ be an optimal cover, and let $x$ be a positive real number smaller than or equal to the minimum of the elements not covered by $c$. Let $A^{\prime}$ be obtained from $A$ by subtracting $x$ from the elements not covered by $c$, and adding $x$ to the doubly covered elements. Then $x$ has been subtracted from the optimal $k$-assignment $k-|c|$ times, and consequently

$$
E(A)=(k-|c|) x+E\left(A^{\prime}\right) .
$$

The previous theorem will be used for random assignment problems, by conditioning on where the minimal non-covered element is.

Theorem 2.5. Suppose $P$ is a standard RAP. Suppose also that a column $c$ is used in every $k-1$-cover of $P$. In particular, this is the case if at least $k$ elements in $c$ are zero. Let $P^{\prime}$ be the RAP with column $c$ deleted from $P$ and $k$ and $n$ decreased by one. Then

$$
E(P)=E\left(P^{\prime}\right) \text {. }
$$

Similarly, if a row belongs to every $k-1$-cover of $P$, it can be deleted. 


\section{Combinatorics of two optimal $k$-Assignments}

In this section we prove some results that are needed in Section 4 . Consider a deterministic assignment problem. If there are two different $k$ assignments $\mu$ and $\nu$, their symmetric difference $\mu \Delta \nu:=(\mu \backslash \nu) \cup(\nu \backslash \mu)$ will be of special importance. By a $(\mu, \nu)$-alternating path we mean a sequence $\left(i_{1}, j_{1}\right),\left(i_{2}, j_{2}\right), \ldots,\left(i_{r}, j_{r}\right)$ of matrix positions, where positions belonging to $\mu$ alternate with positions belonging to $\nu$ and $i_{x}=i_{x+1}$ or $j_{x}=j_{x+1}$, for all $x=1, \ldots, r-1$. The parameter $r$ will be called the length of the path. It is easy to see that the positions in $\mu \Delta \nu$ will form $(\mu, \nu)$-alternating paths in the matrix.

If two $k$-assignments $\mu$ and $\nu$ differ only at zero positions we say that $\mu$ and $\nu$ are equivalent, $\mu \equiv \nu$.

A deterministic assignment problem is semi-generic if there is exactly one nontrivial sum of nonzero elements that equals a sum of a distinct set of nonzero elements.

Lemma 3.1. Let $A$ be a deterministic assignment problem. Let $\mu$ be an optimal k-assignment, and let a be an element which does not belong to $\mu$. Suppose that there is another optimal $k$-assignment $\nu$ that contains a. Then there is an optimal $k$-assignment $\nu^{\prime}$ containing a, such that $\mu \triangle \nu^{\prime}$ consists either of one path, or of two paths of odd length.

Proof. Let $T$ be the path of $\mu \triangle \nu$ that contains $a$. If $T$ is of even length, then we let $\nu^{\prime}=\mu \triangle T$. If $T$ has odd length, then it contains one more element of one of $\mu$ and $\nu$ than of the other. Since $\mu$ and $\nu$ have the same size, there must be another path $T^{\prime}$ of $\mu \triangle \nu$ that balances, so that $T \cup T^{\prime}$ has equally many elements from $\mu$ and $\nu$. Then we let $\nu^{\prime}=\mu \triangle\left(T \cup T^{\prime}\right)$. Clearly $\nu^{\prime}$ has the desired properties.

Lemma 3.2. Let $A$ be a semi-generic matrix with two inequivalent optimal $k$-assignments $\mu$ and $\nu$. Then $\mu \triangle \nu$ has at most two paths with some nonzero element, and if there are two, they both have odd length.

Proof. By Lemma 3.1 there is an optimal $k$-assignment $\nu^{\prime}$ that contains a nonzero element not in $\mu$, such that $\mu \Delta \nu^{\prime}$ has either one path, or two paths of odd length. Since there are only two equivalence classes of optimal $k$-assignments, we must have $\nu^{\prime} \equiv \nu$.

Lemma 3.3. Let $A$ be a deterministic assignment problem. Let $r_{1}$ and $r_{2}$ be rows, and let $c_{1}$ and $c_{2}$ be columns. Suppose that $A\left(r_{1}, c_{1}\right)=0, A\left(r_{2}, c_{1}\right)>0$, and $A\left(r_{2}, c_{2}\right)>0$. Suppose further that there is an optimal $k$-assignment that uses $\left(r_{2}, c_{2}\right)$, but not row $r_{1}$. Then there is no optimal $k$-assignment that uses $\left(r_{2}, c_{1}\right)$.

Proof. Let $\mu$ be an optimal $k$-assignment that uses $\left(r_{2}, c_{2}\right)$, and suppose that there is an optimal $k$-assignment $\nu$ that uses $\left(r_{2}, c_{1}\right)$. By Lemma 3.1] we may assume that $\mu \Delta \nu$ consists of at most two paths, and that if there are two paths, both contain an odd number of matrix elements.

There must be a zero element $\left(s_{1}, c_{1}\right) \in \mu$, otherwise $\left(r_{2}, c_{2}\right)$ could be replaced by $\left(r_{1}, c_{1}\right)$ in $\mu$. Let $S$ be the path of $(\mu \Delta \nu) \backslash\left\{\left(r_{2}, c_{1}\right)\right\}$ that contains $\left(s_{1}, c_{1}\right)$. In other words, $S$ is the set of matrix positions in the 
$(\mu, \nu)$-alternating path that starts at $\left(s_{1}, c_{1}\right)$ and continues in the direction opposite to that of $\left(r_{2}, c_{1}\right)$.

There must be an element $\left(r_{1}, d\right)$ of $\nu$ since otherwise $\left(r_{2}, c_{1}\right)$ could be replaced by $\left(r_{1}, c_{1}\right)$ in $\nu$. Since $\mu$ does not use row $r_{1}$, the element $\left(r_{1}, d\right)$ is in a path of $\mu \Delta \nu$ which is a path with one end belonging to $\nu$. If the other end belongs to $\mu$, the path has an even number of elements, and must therefore be the same as the path containing $\left(r_{2}, c_{1}\right)$. Otherwise the other end too belongs to $\nu$. Then the other path has both ends in $\mu$. In either case, $S$ must end with an element of $\mu$ in a row which is not used by $\nu$.

If the sum of the matrix entries in $\mu \cap S$ is greater than the sum of the matrix entries in $\nu \cap S$, then the cost of the $k$-assignment $(\mu \triangle S) \cup\left\{\left(r_{1}, c_{1}\right)\right\}$ is smaller than the cost of $\mu$, contradicting the optimality of $\mu$. Otherwise the sum of the matrix entries in $\mu \cap S$ is smaller than or equal to the sum of the matrix entries in $\nu \cap S$. Then the cost of $(\nu \triangle S) \backslash\left\{\left(r_{2}, c_{1}\right)\right\}$ is smaller than the cost of $\nu$, again a contradiction.

Lemma 3.4. Let $A$ be a semi-generic deterministic assignment problem, and suppose that there are two non-equivalent optimal $k$-assignments $\mu$ and $\nu$. Assume further that

(1) $\mu$ has a nonzero element in the last row and

(2) $\nu$ does not use the last row.

Then there is a unique row s such that

(1) $\nu$ has a nonzero element in row $s$

(2) There is a k-assignment $\mu^{\prime} \equiv \mu$ that does not use row $s$.

Proof. Existence: Choose a $k$-assignment $\mu^{\prime} \equiv \mu$ which has as many matrix positions as possible in common with $\nu$. Since the last row is used by $\mu^{\prime}$ but not by $\nu$, there has to be a row $s$ which is used by $\nu$ but not by $\mu^{\prime}$. If $\nu$ has a zero element in row $s$, then $\mu^{\prime}$ must contain a zero element in the same column. By replacing this zero by the zero in row $s$, we would obtain a $k$-assignment $\mu^{\prime \prime} \equiv \mu^{\prime}$ which has one more element in common with $\nu$, a contradiction. Therefore, the element in row $s$ which belongs to $\nu$ must be nonzero.

Uniqueness: Assume on the contrary that there are two different rows $s$ and $t$ that contain nonzero elements of $\nu$, say $\left(s, c_{1}\right)$ and $\left(t, c_{2}\right)$, and two equivalent $k$-assignments $\mu \equiv \mu^{\prime}$ such that $\mu$ does not use row $s$ and $\mu^{\prime}$ does not use row $t$. If $\mu$ would not use row $t$ either, then $\mu \Delta \nu$ would have two distinct paths both with one end in a nonzero element of $\nu$, in the elements $\left(s, c_{1}\right)$ and $\left(t, c_{2}\right)$. This contradicts Lemma 3.2

Hence there is a zero element $\left(s, c_{3}\right)$ of $\mu^{\prime}$, and similarly a zero element $\left(t, c_{4}\right)$ of $\mu$. Note that the symmetric difference $\mu \triangle \mu^{\prime}$ contains only zeros and therefore it must consist of a number of $\left(\mu, \mu^{\prime}\right)$-alternating paths, all of even length. Let $U$ be the one that contains $\left(t, c_{4}\right)$. Unless $U$ ends at row $s, \mu \triangle U$ will be a $k$-assignment equivalent to $\mu$ avoiding both rows $s$ and $t$. We have already seen that this is impossible.

We may therefore assume that $\mu \triangle \mu^{\prime}$ consists of a single path from $\left(t, c_{4}\right)$ to $\left(s, c_{3}\right)$. Note that for every row $r$ which is used in this path, there is a $k$-assignment $\mu^{\prime \prime} \equiv \mu$ that avoids row $r$. This is obtained by choosing the 
zeros from $\mu$ in the part of $U$ that goes towards $\left(t, c_{4}\right)$, and choosing them from $\mu^{\prime}$ in the part that goes towards $\left(s, c_{3}\right)$.

Let $(m, d)$ be the position in the last row used by $\mu$ and let $L$ be the $(\mu, \nu)$ alternating path containing $(m, d)$. First note that if $L$ contains $\left(s, c_{1}\right)$, it has to end there and thus be of even length, which means that it has passed through $\left(t, c_{2}\right)$ first.

Case 1. $L$ intersects $U$. In this case, the first element of $L$ (starting from $(m, d))$ that belongs to $U$ must be an element of $\mu$. Suppose that this element is in row $r$. Then let $\mu^{\prime \prime} \equiv \mu$ avoid row $r$. It follows that $\mu^{\prime \prime} \triangle \nu$ contains a path of even length starting at $(m, d)$, and another path containing the nonzero element $\left(s, c_{1}\right)$, contradicting Lemma 3.2

Case 2. $L$ does not intersect $U$. Let $S$ be the path in $\mu \Delta \nu$ containing $\left(s, c_{1}\right),\left(t, c_{4}\right)$ and $\left(t, c_{2}\right)$ in this order.

We know that $U$ and $S$ intersect in $\left(t, c_{4}\right)$. Of the positions in $U \cap S$, let $(r, c)$ be the one which is closest to $\left(s, c_{3}\right)$ in $U$, and let $\mu^{\prime \prime} \equiv \mu$ avoid row $r$. Then $\mu^{\prime \prime} \triangle \nu$ will consist of a cycle containing the nonzero position $\left(s, c_{1}\right)$, and two other paths, one containing $\left(t, c_{2}\right)$ and one containing $(m, d)$, contradicting Lemma 3.2

\section{The probability that a ROW IS USED in the OPtimal ASSignMent}

Crucial for our proof is the following formula for the probability that a certain row without zeros is included in an optimal $k$-assignment. Let $q(P)$ denote this probability.

Theorem 4.1 (Row Inclusion Theorem). Let $P$ be a standard $R A P$, and let $r$ be a row without zeros. The probability that some element in $r$ belongs to the optimal $k$-assignment is

$$
q(P)=\frac{1}{m} \sum_{i} \frac{\overline{d_{i, 0}}(P)}{\left(\begin{array}{c}
m-1 \\
i
\end{array}\right)}
$$

where $\overline{d_{i, 0}}$ is the number of partial $k$-1-covers of $i$ rows not containing the row $r$.

To simplify notation we will assume that the nonzero row in the Row Inclusion Theorem is the last row in the matrix. A consequence of this formula is that the probability of using a certain row without zeros in an optimal $k$-assignment does not change if further zeros are introduced in a row which belongs to an optimal cover. This observation turns out to be sufficient for the proof of the formula.

Lemma 4.2. Let $P$ be a standard $R A P$, and let $r$ be a row that belongs to an optimal cover. Let $P^{\prime}$ be an RAP obtained from $P$ by inserting another zero in row $r$. Suppose there is a row without zeros in $P$. Then

$$
q(P)=q\left(P^{\prime}\right) .
$$

Proof. Suppose that $P$ is a standard RAP where the first row belongs to an optimal cover, and that there is an element in the first row, say $(1,1)$, which is not zero. Suppose further that the last row contains no zeros. We want to show that if we replace the element $(1,1)$ by zero, the probability that the last row is used in the optimal $k$-assignment does not change. 
Let $\Omega_{P}$ be the probability space of all assignments of values to the random variables in $P$, that is, the space of all real nonnegative $m$ by $n$ matrices that have zeros in the positions where $P$ has zeros. If $A \in \Omega_{P}$, we let $q(A)$ be 1 if the last row is used by an optimal $k$-assignment, and 0 otherwise. We let $A_{x}$, for nonnegative real $x$, denote the matrix obtained from $A$ by setting the entry in position $(1,1)$ to $x$.

We construct a measure preserving involution $\varphi$ on $\Omega_{P}$ with the property that (except possibly on a subset of probability zero) if $A \in \Omega_{P}$ is a matrix where the last row changes between being used and not being used in the optimal $k$-assignment when $A(1,1)$ is set to zero, then in $\varphi(A)$, the change goes the other way. In other words,

$$
q(A)-q\left(A_{0}\right)=q\left(\varphi(A)_{0}\right)-q(\varphi(A)) .
$$

Let $A \in \Omega_{P}$. If $q(A)=q\left(A_{0}\right)$, we let $\varphi(A)=A$. Otherwise notice that if we slide the element in position $(1,1)$ continuously down to zero, there can be at most one point at which the location of the optimal $k$-assignment changes, and at this point, the element in position $(1,1)$ goes from not being used to being used.

At the point $A_{f}$ where the change occurs, the matrix is semi-generic with two non-equivalent optimal $k$-assignments. Let $\mu$ be the one that contains an element of the last row, and let $\nu$ be the one that doesn't. By Lemma 3.4. there is a unique row $s$ such that $\nu$ contains a nonzero element in row $s$, and so that there is a $k$-assignment $\mu^{\prime} \equiv \mu$ that contains no element of row $s$. Notice that $s$ cannot be the first row, since the first row is used by every optimal $k$-assignment.

We let $\varphi(A)$ be the matrix obtained from $A$ by swapping the elements in row $s$ with the corresponding elements (elements in the same column) in the last row, except in the columns where row $s$ has zeros.

In the analysis of this mapping, we introduce some auxiliary matrices. Let $A_{f}^{\prime}$ be obtained from $A_{f}$ by setting the elements in the last row that are in columns where row $s$ has zeros, to zero. By Lemma 3.3. we can set these elements as small as we please without changing the location of the optimal assignments. Hence there will be no $k$-assignment of smaller cost than $\mu$ and $\nu$ in $A_{f}^{\prime}$. Then since neither $\mu$ nor $\nu$ uses any zero element in row $s$, these can of course be increased without changing the optimality of $\mu$ and $\nu$. We let $A_{f}^{\prime \prime}$ be the matrix where the zero positions in row $s$ are changed to the values of the corresponding elements in the last row of $A$. If $A$ is generic, every optimal $k$-assignment in $A_{f}^{\prime \prime}$ is equivalent either to $\mu$ or to $\nu$.

Now $\varphi(A)_{f}$ is obtained from $A_{f}^{\prime \prime}$ by swapping row $s$ with the last row. This means that in $\varphi(A)_{f}$, there are exactly two equivalence classes of optimal $k$-assignments, one that includes position $(1,1)$ and one that doesn't. But since the last row has been swapped with another row, we have, for $x \neq f$,

$$
q\left(\varphi(A)_{x}\right)=1-q\left(A_{x}\right),
$$

which implies (5).

In $\varphi(A)_{f}$, row $s$ has the property expressed in the conclusion of Lemma 3.4 but with the roles of $\mu$ and $\nu$ interchanged. It follows that $\varphi(\varphi(A))=A$, and in particular that $\varphi$ is invertible. The mapping $\varphi$ is piecewise linear, 
and on each piece, it is a permutation of variables. Hence it is measure preserving. This completes the proof.

Proof of Theorem 4.1. We now establish the Row Inclusion Theorem by an inductive argument. Let $P$ be a standard RAP, and suppose the last row contains no zero element. Notice that by König's theorem (see LP86), the formula holds whenever $P$ has a $k$-assignment of only zeros. Suppose that the formula has been established for every standard RAP with fewer nonzero elements than $P$.

Case 1: Some optimal cover of $P$ contains a row $r$ with at least one nonzero element. Then every optimal $k$-assignment must use row $r$. Let $P^{\prime}$ be as $P$ but with another zero in row $r$. By Theorem 4.2, the probability that the last row is used is the same in $P$ as in $P^{\prime}$. Hence we only have to show that $\overline{d_{i, 0}}(P)=\overline{d_{i, 0}}\left(P^{\prime}\right)$ for $i=0, \ldots, k-1$. We have to show that if a set of rows can be extended to a $k$-1-cover of $P$, then it is possible to use row $r$ in this $k-1$-cover. This will follow if we can show that row $r$ belongs to the optimal cover of the remaining zeros.

It suffices to show that if a row $s \neq r$ is deleted from the matrix, row $r$ still belongs to the optimal cover of the remaining zeros. If the deletion of $s$ does not decrease the maximal number of independent zeros, this is obvious. Suppose therefore that the deletion of $s$ decreases the number of independent zeros. Then $s$ belongs to an optimal cover of $P$. Hence the rowmaximal optimal cover of $P$ contains both $r$ and $s$. When row $s$ is deleted, the remaining rows and columns including $r$ will constitute an optimal cover of the remaining zeros.

Case 2: There is a row in $P$ with only zeros. Let $P^{\prime}$ be obtained from $P$ by deleting this row and decreasing $k$ and $m$ by 1 . The probability that the last row is used by an optimal assignment is clearly the same in $P$ as in $P^{\prime}$. By induction, the probability that the last row is used in $P^{\prime}$ is (with $k=k(P), m=m(P))$

$$
\frac{1}{m-1} \sum_{i=0}^{k-2} \frac{\overline{d_{i, 0}}\left(P^{\prime}\right)}{\left(\begin{array}{c}
m-2 \\
i
\end{array}\right)} .
$$

We have

$$
\overline{d_{i, 0}}(P)=\overline{d_{i, 0}}\left(P^{\prime}\right)+\overline{d_{i-1,0}}\left(P^{\prime}\right)
$$

for every $i$. Hence

$$
\text { (6) } \begin{aligned}
\frac{1}{m} \sum_{i} \frac{\overline{d_{i, 0}}(P)}{\left(\begin{array}{c}
m-1 \\
i
\end{array}\right)} & =\frac{1}{m} \sum_{i=0}^{k-1} \frac{\overline{d_{i, 0}}\left(P^{\prime}\right)+\overline{d_{i-1,0}}\left(P^{\prime}\right)}{\left(\begin{array}{c}
m-1 \\
i
\end{array}\right)} \\
& =\frac{1}{m} \sum_{i} \frac{\overline{d_{i, 0}}\left(P^{\prime}\right)}{\left(\begin{array}{c}
m-1 \\
i
\end{array}\right)}+\frac{1}{m} \sum_{i} \frac{\overline{d_{i, 0}}\left(P^{\prime}\right)}{\left(\begin{array}{c}
m-1 \\
i+1
\end{array}\right)} \\
& =\sum_{i} \overline{d_{i, 0}}\left(P^{\prime}\right)\left(\frac{1}{m\left(\begin{array}{c}
m-1 \\
i
\end{array}\right)}+\frac{1}{m\left(\begin{array}{c}
m-1 \\
i+1
\end{array}\right)}\right)=\frac{1}{m-1} \sum_{i} \frac{\overline{d_{i .0}}\left(P^{\prime}\right)}{\left(\begin{array}{c}
m-2 \\
i
\end{array}\right)} .
\end{aligned}
$$

Case 3: There is a unique optimal cover $c$ consisting of only columns. Then these columns will be used by the optimal $k$-assignment. Therefore the number of elements not covered by $c$ in the optimal $k$-assignment is independent of the random variables in the matrix. We condition on the 
position of the minimal element not covered by $c$. If in each case we subtract this minimum from all elements not covered by $c$, the same nonzero elements will be used in the optimal $k$-assignments. Here we are using Theorem 2.4 in the special case of no doubly covered elements. Let $P^{t}$ be the RAP obtained by conditioning on the minimal element in $P$ not covered by $c$ being in row $t$, and subtracting this minimum from all elements not covered by $c$. Then $P^{t}$ is a standard RAP, and the new zero occurring in row $t$ means that row $t$ belongs to an optimal cover of $P^{t}$. Hence in case the new zero is in the last row, that row must be used in the optimal $k$-assignment, while if it is not, we can find the probability that the last row is used by induction. We let $P \backslash t$ be the RAP obtained by deleting row $t$ from $P$ and decreasing $k$ by 1. Case 1 and 2 implies that the probability that the last row is used is the same in $P^{t}$ as in $P \backslash t$. It follows that the probability that the last row is used in the optimal $k$-assignment in $P$ is given by

$$
\frac{1}{m}+\frac{1}{m(m-1)} \sum_{t} \sum_{i} \frac{\overline{d_{i, 0}}(P \backslash t)}{\left(\begin{array}{c}
m-2 \\
i
\end{array}\right)} .
$$

Here we have

$$
\sum_{t} \overline{d_{i, 0}}(P \backslash t)=(i+1) \overline{d_{i+1,0}}(P),
$$

since both sides are equal to the sum, taken over all $t$, of the number of partial $k$-1-covers of $P$ that use row $t$ (and not the last row). It follows that (17) equals

$$
\text { (8) } \begin{aligned}
& \frac{1}{m}+\frac{1}{m(m-1)} \sum_{i} \frac{(i+1) \overline{d_{i+1,0}}(P)}{\left(\begin{array}{c}
m-2 \\
i
\end{array}\right)} \\
&=\frac{1}{m}+\frac{1}{m} \sum_{i} \frac{\overline{d_{i+1,0}}(P)}{\left(\begin{array}{c}
m-1 \\
i+1
\end{array}\right)}=\frac{1}{m} \sum_{i} \frac{\overline{d_{i, 0}}(P)}{\left(\begin{array}{c}
m-1 \\
i
\end{array}\right)} .
\end{aligned}
$$

This completes the proof of Theorem 4.1

\section{Proof of the COVER FORMUla For LARGE $m$ OR LARGE $n$}

The results of the last section will now enable us to prove that the cover formula (2) holds for standard RAP's whenever either $m$ or $n$ is sufficiently large compared to $k$. We first prove that the cover formula is consistent with the Row Inclusion Theorem.

Theorem 5.1. Let $P$ be a standard $R A P$ where the last row contains no zeros, and let $P_{t}$ be obtained from $P$ by setting the element in column $t$ of the last row to zero. If the cover formula (2) holds for every $P_{t}$, then it holds for $P$.

Proof. By Theorem 2.2 the probability that the element in column $t$ in the last row belongs to an optimal $k$-assignment in $P$ is equal to $E(P)-E\left(P_{t}\right)$. Hence

$$
n E(P)-\sum_{t} E\left(P_{t}\right)=\frac{1}{m} \sum_{i} \frac{\overline{d_{i, 0}}(P)}{\left(\begin{array}{c}
m-1 \\
i
\end{array}\right)} .
$$

Suppose that the cover formula holds for each $P_{t}$. Then 


$$
n E(P)=\frac{1}{m n} \sum_{t} \sum_{i, j} \frac{d_{i, j}\left(P_{t}\right)}{\left(\begin{array}{c}
m-1 \\
i
\end{array}\right)\left(\begin{array}{c}
n-1 \\
j
\end{array}\right)}+\frac{1}{m} \sum_{i} \frac{\overline{d_{i, 0}}(P)}{\left(\begin{array}{c}
m-1 \\
i
\end{array}\right)} .
$$

In order to prove that the cover formula holds for $P$, it is sufficient to prove that

$$
\sum_{i, j} \frac{d_{i, j}(P)}{\left(\begin{array}{c}
m-1 \\
i
\end{array}\right)\left(\begin{array}{c}
n-1 \\
j
\end{array}\right)}=\frac{1}{n} \sum_{t} \sum_{i, j} \frac{d_{i, j}\left(P_{t}\right)}{\left(\begin{array}{c}
m-1 \\
i
\end{array}\right)\left(\begin{array}{c}
n-1 \\
j
\end{array}\right)}+\sum_{i} \frac{\overline{d_{i, 0}}(P)}{\left(\begin{array}{c}
m-1 \\
i
\end{array}\right)}
$$

If we write

$$
\overline{d_{i, 0}}(P)=\sum_{j}\left(\frac{\overline{d_{i, j}}(P)}{\left(\begin{array}{l}
n \\
j
\end{array}\right)}-\frac{\overline{d_{i, j+1}}(P)}{\left(\begin{array}{c}
n \\
j+1
\end{array}\right)}\right),
$$

and fix $i$ and $j$, we see that (9) will follow from the identity

$$
\frac{d_{i, j}(P)}{\left(\begin{array}{c}
n-1 \\
j
\end{array}\right)}=\frac{\overline{d_{i, j}}(P)}{\left(\begin{array}{c}
n \\
j
\end{array}\right)}-\frac{\overline{d_{i, j+1}}(P)}{\left(\begin{array}{c}
n \\
j+1
\end{array}\right)}+\frac{1}{n\left(\begin{array}{c}
n-1 \\
j
\end{array}\right)} \sum_{t} d_{i, j}\left(P_{t}\right) .
$$

Here, partial covers that contain the last row will contribute $1 /\left(\begin{array}{c}n-1 \\ j\end{array}\right)$ to both sides. If we let $\overline{d_{i, j}}\left(P_{t}\right)$ denote the number of partial $k$-1-covers of $P_{t}$ with $i$ rows and $j$ columns that do not contain the last row, it only remains to show that

or equivalently

$$
\frac{\overline{d_{i, j}}(P)}{\left(\begin{array}{c}
n-1 \\
j
\end{array}\right)}=\frac{\overline{d_{i, j}}(P)}{\left(\begin{array}{c}
n \\
j
\end{array}\right)}-\frac{\overline{d_{i, j+1}}(P)}{\left(\begin{array}{c}
n \\
j+1
\end{array}\right)}+\frac{1}{n\left(\begin{array}{c}
n-1 \\
j
\end{array}\right)} \sum_{t} \overline{d_{i, j}}\left(P_{t}\right),
$$

$$
j \overline{d_{i, j}}(P)+(j+1) \overline{d_{i, j+1}}(P)=\sum_{t} \overline{d_{i, j}}\left(P_{t}\right) .
$$

Here the first term of the left hand side counts the partial $k$-1-covers of $P_{t}$ that contain column $t$, while the second term counts those that don't. Hence (9) holds.

Next we show that the cover formula is consistent with removing a column which belongs to every $k-1$-cover.

Theorem 5.2. Let $P$ be a standard $R A P$, and suppose that the first column belongs to every $k-1$-cover. Let $P^{\prime}$ be the RAP obtained from $P$ by deleting the first column and decreasing $k$ and $n$ by 1 . If the cover formula holds for $P^{\prime}$, then it holds for $P$.

Proof. By Theorem 2.5 $E(P)=E\left(P^{\prime}\right)$. We have (with $n=n(P)$ and $k=k(P))$

$$
\begin{aligned}
& \frac{1}{m n} \sum_{i, j} \frac{d_{i, j}(P)}{\left(\begin{array}{c}
m-1 \\
i
\end{array}\right)\left(\begin{array}{c}
n-1 \\
j
\end{array}\right)}=\frac{1}{m n} \sum_{i, j} \frac{d_{i, j-1}\left(P^{\prime}\right)+d_{i, j}\left(P^{\prime}\right)}{\left(\begin{array}{c}
m-1 \\
i
\end{array}\right)\left(\begin{array}{c}
n-1 \\
j
\end{array}\right)} \\
= & \frac{1}{m n} \sum_{i, j}\left(\frac{d_{i, j}\left(P^{\prime}\right)}{\left(\begin{array}{c}
m-1 \\
i
\end{array}\right)\left(\begin{array}{c}
n-1 \\
j
\end{array}\right)}+\frac{d_{i, j}\left(P^{\prime}\right)}{\left(\begin{array}{c}
m-1 \\
i
\end{array}\right)\left(\begin{array}{c}
n-1 \\
j+1
\end{array}\right)}\right)=\frac{1}{m(n-1)} \sum_{i, j} \frac{d_{i, j}\left(P^{\prime}\right)}{\left(\begin{array}{c}
m-1 \\
i
\end{array}\right)\left(\begin{array}{c}
n-2 \\
j
\end{array}\right)} \\
& =E\left(P^{\prime}\right)=E(P) .
\end{aligned}
$$


Theorem 5.3. If $P$ is a standard $R A P$ such that $\max (m, n)>(k-1)^{2}$, then

$$
E(P)=\frac{1}{m n} \sum_{i, j} \frac{d_{i, j}(P)}{\left(\begin{array}{c}
m-1 \\
i
\end{array}\right)\left(\begin{array}{c}
n-1 \\
j
\end{array}\right)} .
$$

Proof. We show that if $m>(k-1)^{2}$, then a standard RAP which contains a zero in each row must have a column which belongs to every $k-1$-cover. The theorem then follows by induction on the number of nonzero elements of $P$. It suffices to consider the case that $P$ has exactly one zero in each row. Then the total number of zeros is greater than $(k-1)^{2}$. Either there is a column with $k$ or more zeros, which must then belong to every $k-1$ cover, or there is an independent set of $k$ zeros, and in this case there is no $k-1$-cover.

By symmetry, the formula also holds if $n>(k-1)^{2}$.

\section{Rationality of $E(P)$ as a Function of $n$}

We know from Section 5 that for fixed $k$, whenever $m$ or $n$ is large, the cover formula (2) for standard RAP's holds. In order to prove that the formula holds for smaller values of $m$ and $n$, it is therefore sufficient to show that if $k, m$ and the zero positions are fixed, and we let $P_{n}$ be the standard RAP with $n$ columns, then there is a rational function in the variable $n$ that gives the value of $E\left(P_{n}\right)$ for every $n$ which is at least as large as $k$ and the number of columns with zeros. This rational function must then be equal to the one given by the cover formula. We prove this by induction over a class of RAP's which includes not only standard RAP's.

In an exponential RAP, all matrix elements are linear combinations with nonnegative rational coefficients of a set $X_{1}, \ldots, X_{p}$ of independent exponentially distributed random variables. If a variable $X_{i}$ in an exponential RAP has intensity 1 , and occurs in one and only one matrix position, and this matrix entry is equal to $X_{i}$, then the variable is called a standard variable, and the matrix position where it occurs is called a standard position.

We introduce the concept of an RAP-sequence. The idea is to treat a set of similar RAP's with different number of columns in a uniform way, in order to prove that there is a rational expression in the number of columns that gives the value of each RAP in the set.

Definition 6.1. We say that a linear function $f(x)=a x+b$ in one variable is $k$-positive, iff $f(x)>0$ whenever $x \geq k$, or equivalently, if $a \geq 0$ and $b>-a k$.

Obviously, the sum of two or more $k$-positive functions is $k$-positive.

Definition 6.2. An $R A P$-sequence is a sequence $P_{n}\left(n \geq n_{0}\right)$ of exponential RAP's satisfying the following:

(1) Each $P_{n}$ is an exponential RAP in a set of variables $X_{n, 1}, \ldots, X_{n, p(n)}$.

(2) The numbers $m$ and $k$ are uniform, that is $m\left(P_{i}\right)=m\left(P_{j}\right)$ and $k\left(P_{i}\right)=k\left(P_{j}\right)$ for all $i$ and $j$, while $P_{n}$ has $n$ columns. 
(3) In the first $n_{0}$ columns, the matrix elements of the various RAP's differ only in that the first index of the variables is changed. In other words, the coefficient of $X_{n, i}$ in a matrix element in the first $n_{0}$ columns of $P_{n}$ is equal to the coefficient of $X_{n_{0}, i}$ in the same position in $P_{n_{0}}$.

(4) Beyond column $n_{0}, P_{n}$ has only standard elements.

Moreover, we say that the RAP-sequence is well-behaved if

(1) For each $i$ such that the variables $X_{n, i}$ occur in the first $n_{0}$ columns, there is a $k$-positive linear polynomial $f_{i}(n)$ such that the intensity of $X_{n, i}$ is $f_{i}(n)$.

(2) Nonzero nonstandard elements occur only in columns which belong to the column-maximal optimal cover of the zeros, or equivalently, columns that intersect every maximal set of independent zeros.

Theorem 6.3 (Rationality Theorem). Suppose $P$ is a well-behaved RAPsequence. Then there is a rational function $f(x)$ in one variable such that

(1) If $x$ is a zero of the denominator of $f$, then $x<k$.

(2) $E\left(P_{n}\right)=f(n)$ for every $n \geq n_{0}(P)$.

Definition 6.4. We say that a linear combination $u_{1}$ of variables $X_{1}, \ldots, X_{p}$ is smaller than another if for each $X_{i}$, the coefficient is smaller in $u_{1}$ than in $u_{2}$. We say that $u_{1}$ and $u_{2}$ are incomparable if neither of them is smaller than the other, in other words, if each of them has a higher coefficient than the other for some variable.

We say that a linear combination is potentially minimal in a set of linear combinations, if it is smaller than or incomparable with every other.

Let $c$ be the row-maximal optimal cover of $P$. We prove the rationality theorem by induction on a number of parameters, in the following order:

(1) The size of the largest independent set of zeros. An RAP-sequence is considered simpler if it has a larger set of independent zeros.

(2) The number of rows in $c$. If the number of independent zeros are equal, the RAP-sequence with fewer rows belonging to the rowmaximal optimal cover is simpler.

(3) The set of potentially minimal nonstandard elements not covered by c. If 1 . and 2. are equal, an RAP-sequence is considered simpler if fewer of the nonstandard elements not covered by $c$ are potentially minimal.

(4) If 1-3 are equal, and there are two incomparable nonstandard elements not covered by $c$, then an RAP-sequence is simpler if there are fewer variables with different coefficients in the first two (in lexicographic order, say) incomparable potentially minimal nonstandard elements not covered by $c$.

(5) If 1-3 are equal, and there is a minimal non-covered nonstandard element, then an RAP-sequence is simpler if the number of variables occurring in this element is smaller.

Let $P$ be a well-behaved RAP-sequence, and suppose that the rationality theorem holds for every simpler well-behaved RAP-sequence with the same values of $m$ and $k$. We may of course assume that $|c|<k$. Since $P$ is 
well-behaved, in each row all but at most $k-1$ elements are standard and not covered by $c$.

We show that $E(P)$ can be expressed in terms of rational functions in $n$, and values of simpler well-behaved RAP-sequences.

Case 1: There are two or more non-covered incomparable nonstandard elements. Let $u_{1}$ and $u_{2}$ be the first two (in lexicographic order). We choose $i$ and $j$ such that the coefficient of $X_{n, i}$ is greater in $u_{1}$, and the coefficient of $X_{n, j}$ is greater in $u_{2}$. Let the coefficients be $a_{1}, a_{2}, b_{1}$ and $b_{2}$ so that $u_{1}=a_{1} X_{n, i}+b_{1} X_{n, j}+\ldots$ and $u_{2}=a_{2} X_{n, i}+b_{2} X_{n, j}+\ldots$

Let $Q$ and $R$ be the RAP-sequences obtained by conditioning on $\left(a_{1}-\right.$ $\left.a_{2}\right) X_{n, i}$ being smaller or greater than $\left(b_{2}-b_{1}\right) X_{n, j}$, respectively.

The intensities of $\left(a_{1}-a_{2}\right) X_{n, i}$ and $\left(b_{2}-b_{1}\right) X_{n, j}$ are $f_{i}(n) /\left(a_{1}-a_{2}\right)$ and $f_{j}(n) /\left(b_{2}-b_{1}\right)$ respectively. The probability of $\left(a_{1}-a_{2}\right) X_{n, i}$ being smaller than $\left(b_{2}-b_{1}\right) X_{n, j}$ is

$$
\frac{f_{i}(n) /\left(a_{1}-a_{2}\right)}{f_{i}(n) /\left(a_{1}-a_{2}\right)+f_{j}(n) /\left(b_{2}-b_{1}\right)},
$$

and similarly, the probability of $\left(b_{2}-b_{1}\right) X_{n, j}$ being smaller than $\left(a_{1}-a_{2}\right) X_{n, i}$ is

Therefore,

$$
\frac{f_{j}(n) /\left(b_{2}-b_{1}\right)}{f_{i}(n) /\left(a_{1}-a_{2}\right)+f_{j}(n) /\left(b_{2}-b_{1}\right)} .
$$

$$
E\left(P_{n}\right)=\frac{f_{i}(n) E\left(Q_{n}\right) /\left(a_{1}-a_{2}\right)+f_{j}(n) E\left(R_{n}\right) /\left(b_{2}-b_{1}\right)}{f_{i}(n) /\left(a_{1}-a_{2}\right)+f_{j}(n) /\left(b_{2}-b_{1}\right)} .
$$

We show that $Q$ and $R$ can be regarded as well-behaved RAP-sequences. If we condition on $\left(a_{1}-a_{2}\right) X_{n, i}$ being smaller than $\left(b_{2}-b_{1}\right) X_{n, j}$, then we can write

$$
\left(a_{1}-a_{2}\right) X_{n, i}=Y_{n}
$$

and

$$
\left(b_{2}-b_{1}\right) X_{n, j}=Y_{n}+Z_{n},
$$

where $Y_{n}$ and $Z_{n}$ are independent exponentially distributed variables. The intensities are given by

$$
I\left(Y_{n}\right)=\frac{f_{i}(n)}{a_{1}-a_{2}}+\frac{f_{j}(n)}{b_{2}-b_{1}}
$$

and

both of which are $k$-positive.

$$
I\left(Z_{n}\right)=\frac{f_{j}(n)}{b_{2}-b_{1}}
$$

When replacing $X_{n, i}$ and $X_{n, j}$ by the new variables $Y_{n}$ and $Z_{n}$, only the nonstandard elements are affected. Either $u_{1}$ is smaller than $u_{2}$ in $Q$, or at least the number of variables with distinct coefficients is smaller than in $P$, since $u_{1}$ and $u_{2}$ will get the same coefficient for $Y_{n}$. For any two matrix elements that satisfied $u_{3} \leq u_{4}$ before we conditioned on which one of $X_{n, i}$ and $X_{n, j}$ is smallest, this inequality will still hold.

Hence $Q$, and similarly $R$, are simpler than $P$. By induction, it follows that (11) gives a rational expression for $E(P)$, where the denominator is nonzero for $n \geq k$. 
Case 2: There are no two non-covered incomparable nonstandard elements. Then either there is among the non-covered nonstandard elements a minimal one, or there are no non-covered nonstandard elements. We can treat these slightly different cases in the same way.

If there is a minimal non-covered nonstandard element, let $a X_{n, i}$ be a term occurring in this matrix entry. We let $S_{n}$ be a set of random variables consisting of $a X_{n, i}$ and all the non-covered standard variables (if there is no non-covered nonstandard element, we let $S_{n}$ consist only of the non-covered standard elements). There are at least $n-k+1$ non-covered standard elements in each non-covered row. By grouping together the standard elements in each row, we can write the total intensity of $S_{n}$ in a uniform way as a sum of $k$-positive terms. Therefore the intensity of the minimum $Y_{n}$ of the terms in $S_{n}$ is $k$-positive as a function of $n$.

We condition on the minimal element in $S_{n}$. We can then replace the terms in $S_{n}$ by new variables $Y_{n}$ and $Z_{n, i}$ where $Y_{n}=\min \left(S_{n}\right)$ and $Z_{n, i}$ are the differences between the remaining terms in $S_{n}$ and the minimum.

Since all non-covered nonstandard elements contain the variable $X_{n, i}$ with a coefficient of at least $a$, we can by Theorem 2.4 subtract the minimum of $S_{n}$ from every non-covered element, and add it to the doubly covered elements.

We then get

$$
E\left(P_{n}\right)=\frac{k-|c|}{I\left(Y_{n}\right)}+\frac{1}{I\left(Y_{n}\right)} \sum_{t \in S_{n}} I(t) E\left(Q_{n}(t)\right),
$$

where $Q_{n}(t)$ are the new RAP's obtained by conditioning on the term $t$ being smallest, and performing the change of variables and subtraction of the minimum, and $I(t) / I\left(Y_{n}\right)$ is the corresponding probability. In order to write this in a uniform way for the different values of $n$, we group together the cases where the minimum occurs in a particular row and beyond column $n_{0}$.

By permuting columns, we may assume that in those cases, the minimum always occurs in column $n_{0}+1$. The probability for each such case is $(n-$ $\left.n_{0}\right) / I\left(Y_{n}\right)$. In this way, (12) will contain the same terms for each $n>n_{0}$.

If we let $S_{n}^{\prime}$ be the subset of $S_{n}$ consisting of variables occurring in the first $n_{0}$ columns, and we let $S_{n}^{\prime \prime}$ be the set of standard variables in column $n_{0}+1$, we get

$$
E\left(P_{n}\right)=\frac{k-|c|}{I\left(Y_{n}\right)}+\frac{1}{I\left(Y_{n}\right)} \sum_{t \in S_{n}^{\prime}} I(t) E\left(Q_{n}(t)\right)+\frac{n-n_{0}}{I\left(Y_{n}\right)} \sum_{t \in S_{n}^{\prime \prime}} I(t) E\left(Q_{n}(t)\right) .
$$

For $n=n_{0}$, the second sum will be empty. However, provided we can show that $Q(t)$ is a well-behaved RAP-sequence simpler than $P$, it will follow by induction that the denominator of $E(Q(t))$ does not vanish for $n \leq k$. Hence the rational expression that occurs when multiplying the probability $\left(n-n_{0}\right) / I\left(S_{n}\right)$ with the expression for $E(Q(t))$ will vanish for $n=n_{0}$. Therefore the rational expression that results will give the correct value of $E(P)$ also for $n=n_{0}$.

It remains to show that $Q(t)$ is a well-behaved RAP-sequence, and that it is simpler than $P$. We first show that $Q(t)$ is well-behaved. We have already 
seen that the intensities of the variables occurring in $Q(t)$ are $k$-positive. We therefore turn to the distribution of nonstandard elements. Possibly, there are some new nonstandard elements among the doubly covered elements. If there is no new zero among the non-covered elements, the columns of the doubly covered positions of course belong to the column-maximal optimal cover. Suppose that a new zero occurs among the non-covered elements. If the new zero is in a column that belongs to the column-maximal cover of $P$, then this is still the column-maximal cover of the new set of zeros. If the new zero is in a column that does not belong to the column-maximal cover, then since it is also in a row that does not belong to the row-maximal cover, there must be an independent set of zeros in $Q(t)$ which is larger than the largest independent set of zeros in $P$. Therefore, the column-maximal cover in $P$, extended with the column where the new zero has occurred, will be an optimal cover in $Q(t)$. Hence in any case, the columns of the column-maximal cover in $P$ belong to the column-maximal cover of $Q(t)$.

We now show that $Q(t)$ is simpler than $P$. If $Q(t)$ contains a new zero, then either it gives a larger independent set of zeros, or it has to be covered by a column in the row-maximal cover in $Q(t)$. In either case, $Q(t)$ is simpler than $P$. If no new zero occurs, this must be because the minimal term in $S_{n}$ was a term occurring in the minimal non-covered nonstandard element. In this case, the number of variables in this element will decrease, again making $Q(t)$ simpler than $P$.

Hence (12) gives a rational expression for $E(P)$ whose denominator is non-vanishing for $n \geq k$.

This completes the proof of Theorem 6.3

We are finally able to give a proof of Theorem 1.3 which we restate.

Theorem 1.3 If $P$ is a standard RAP, then

$$
E(P)=\frac{1}{m n} \sum_{i, j} \frac{d_{i, j}(P)}{\left(\begin{array}{c}
m-1 \\
i
\end{array}\right)\left(\begin{array}{c}
n-1 \\
j
\end{array}\right)} .
$$

Proof. A standard RAP with $n_{0}$ columns can be extended to a well-behaved RAP-sequence $P_{n}$ by inserting more columns without zeros. Hence Theorem 6.3 shows that there is a rational function $f$ giving the expected value of $P_{n}$. Since the cover coefficient $d_{i, j}\left(P_{n}\right)$ can be expressed uniformly as a polynomial in $n$, the cover formula gives a rational function in $n$ which takes the same values as $f$ on the infinitely many integers $n>\max \left(n_{0},(k-1)^{2}\right)$. Hence the cover formula must agree with $f$, and give the value of $E(P)$.

\section{Asymptotic Results}

Besides giving a new proof of Aldous' $\zeta(2)$-limit theorem, Theorem 1.3 also makes the conjectured limits of [LW00] rigorous. It follows already

from the conjecture of Coppersmith and Sorkin, now Theorem 1.2 that if $\alpha, \beta \geq 1$, then as $k \rightarrow \infty$, the value of the optimal $k$-assignment in a $[\alpha k]$ by $[\beta k]$-matrix of $\exp (1)$-variables converges to

$$
\int_{\Delta} \frac{d x d y}{(\alpha-x)(\beta-y)}
$$


where $\Delta$ is the triangle with vertices in $(0,0),(1,0)$ and $(0,1)$. For instance, when $\alpha=1$ and $\beta=2$ the limit is equal to

$$
\frac{\pi^{2}}{12}-\frac{(\log 2)^{2}}{2}
$$

It also follows that the value of a standard RAP with zeros in a region which is scaled up with $k, m$ and $n$ will converge to a similar integral. In particular it is shown in LW00 that the cover formula implies that the limit value of a standard square RAP with zeros outside an inscribed circle is equal to $\pi^{2} / 24$.

A result presented as a conjecture in O92, and proved in A01, states that in the case $k=m=n$ with no zeros, as $n \rightarrow \infty$, the probability that the smallest element in a row belongs to the optimal assignment converges to $1 / 2$. We can now give an exact formula for this probability for finite $k$,

$$
\frac{1}{2}+\frac{1}{2 k} \text {. }
$$

In the case $k=m$, the probability that the smallest element in a particular row belongs to the optimal assignment is equal to the probability that the smallest element in the entire matrix does. For arbitrary $k, m$, and $n$, the probability that the smallest element in the matrix belongs to the optimal $k$-assignment is

$$
1-\frac{k(k-1)}{2 m n}
$$

These theorems are obtained by combining the results of [LW00] and Theorem 1.3 .

\section{REFERENCES}

[A92] Aldous, D., Asymptotics in the random assignment problem, Pr. Th. Related Fields, 93 (1992) 507-534.

[A01] Aldous, D., The $\zeta(2)$ limit in the random assignment problem, Random Structures Algorithms 18 (2001), no 4. 381-418.

[AS02] Alm, Sven-Erick and Sorkin, Gregory B, Exact expectations and distributions in the random assignment problem, Combin. Probab. Comput. 11 (2002), no. 3, 217-248.

[BFM98] Beveridge Andrew, Frieze, Alan M. and McDarmid Colin J.H., Random minimum length spanning trees in regular graphs, Combinatorica 18 (1998) 311-333.

[BCR02] Buck, M. W., Chan, C. S., Robbins, D. P., On the expected value of the minimum assignment, Random Structures Algorithms 21 (2002), no. 1, 33-58.

[CS98] Coppersmith, D., Sorkin, G. B., Constructive Bounds and Exact Expectations For the Random Assignment Problem, Random Structures Algorithms 15 (1999), 133-144.

[CS02] Coppersmith, D., Sorkin, G. B., On the expected incremental cost of a minimum assignment. In Contemporary Mathematics (B. Bollobás, ed.), Vol. 10 of Bolyai Society Mathematical Studies, Springer.

[D69] Donath, W. E., Algorithm and average-value bounds for assignment problems, IBM J. Res. Dev., 13 (1969) 380-386.

[EES01] Eriksson, H., Eriksson, K., and Sjöstrand, J., Exact expectation for random graphs and assignments, Proceedings of FPSAC 2001, Arizona.

[FM89] Frieze, Alan M. and McDarmid Colin J.H., On random minimum spanning trees, Combinatorica 9 (1989).

[F85] Frieze, Alan M., On the value of a random minimum spanning tree problem, Disc Applied Math. 10 (1985) 47-56. 
[GK93] Goemans, M. X., and Kodialam, M. S., A lower bound on the expected cost of an optimal assignment, Math. Oper. Res., 18 (1993), 267-274.

[K87] Karp, R. M., An upper bound on the expected cost of an optimal assignment, In Discrete Algorithms and Complexity: Proceedings of the Japan-U.S. Joint Seminar, Academic Press, 1987, 1-4.

[L93] Lazarus, A., Certain expected values in the random assignment problem, Oper. Res. Lett., 14 (1993), 207-214.

[LW00] Linusson, S., and Wästlund, J., A generalization of the random assignment problem, arXiv:math.CO/0006146

[LP86] Lovász, L., Plummer, M. D., Matching Theory, North-Holland 1986.

[MP85] Mézard, M. and Parisi, G., Replicas and optimization, J. Phys. Lett. 46(1985), 771-778.

[MP87] Mézard, M. and Parisi, G., On the solution of the random link matching problems, J. Phys. Lett. 48 (1987), 1451-1459.

[O92] Olin, Birgitta, Asymptotic properties of the random assignment problem, Ph.D. thesis, Kungl Tekniska Högskolan, Stockholm, Sweden, (1992).

[PR93] Pardalos, P. M. and Ramakrishnan, K. G., On the expected optimal value of random assignment problems: Experimental results and open questions. Comput. Optim. Appl. 2 (1993), 261-271.

[P98] Parisi, Giorgio, A conjecture on random bipartite matching, Physics e-Print archive, http://xxx.lanl.gov/ps/cond-mat/9801176 January (1998).

[W79] Walkup, D. W., On the expected value of a random assignment problem, SIAM J. Comput., 8 (1979), 440-442.

Svante Linusson, Department of Mathematics, Linköpings universitet, SE58183 LinkÖPING, SWEDEN

E-mail address: linusson@mai.liu.se

Johan Wästlund, Department of Mathematics, LinkÖpings universitet, SE58183 LinkÖPING, SwEDEN

E-mail address: jowas@mai.liu.se 